\title{
UNA PROPUESTA DE INNOVACIÓN EN LA FORMACIÓN UNIVERSITARIA: E - LEARNING COMO APOYO AL APRENDIZAJE REFLEXIVO
}

\author{
Laura RAYÓN RUMAYOR1 \\ Carmen ALCALDE SPIRITO2 \\ Ana de la HERAS CUENCA3 \\ Juan GUTIÉRREZ GARCÍA4
}

\begin{abstract}
RESUMEN: Presentamos una propuesta de innovación5 en la formación del profesorado basada en la implantación del e-learning como apoyo a un aprendizaje reflexivo. El contexto de aplicación se centra en las Prácticas de Enseñanza, concretamente en la asignatura Practicum de los estudios de Magisterio, materia que da cobertura a un aprendizaje basado en una reflexión crítica sobre la práctica educativa en los centros escolares. Las asignaturas implicadas han sido dos, el Practicum I y el Practicum II, y los alumnos implicados han sido treinta y cuatro. En este documento presentamos el marco teórico y los principios rectores que han guiado el diseño y desarrollo de la experiencia. Nos detenemos también en los datos recogidos durante el proceso y los resultados obtenidos en relación a las implicaciones que tiene todo ello en la práctica educativa a partir del análisis de nuestras prácticas de enseñanza y de aprendizaje. Con ello queremos evidenciar las posibilidades didácticas del e-learning y sus beneficios para favorecer procesos de aprendizaje reflexivos y críticos en la formación del profesorado.
\end{abstract}

PALABRAS CLAVE: E-learning. Formación del profesorado. Prácticas de enseñanza. Aprendizaje para la comprensión. Enseñanza reflexiva.

Todo lo que nos es familiar, tiende a ser apreciado como natural; cuando eso ocurre le damos carta de naturaleza a lo que nos rodea, a los contactos y a la relaciones que mantenemos con lo que nos circunda, como si su

existencia fuera resultado de la espontaneidad, como si siempre hubiera existido e inevitablemente tuviera que existir. Esa habituación cala en nuestras vidas, da sentido a cómo entendemos y nos representamos el mundo de la cotidianeidad; es decir, da contenido a nuestro sentido común. Éste nos sirve para percibir y dar significado a la realidad natural, social y humana José Gimeno Sacristán (2003, p.45).

\footnotetext{
1 Coordinadora del proyecto de Innovación. Universidade de Alcalá. Departamento Didáctica. Alcalá de Henares - Madrid - España. laura.rayon@uah.es

2 Universidade de Alcalá. Departamento Didáctica. Alcalá de Henares - Madrid - España.

3 Becaria Investigación. Universidade de Alcalá. Departamento Didáctica. Alcalá de Henares - Madrid - España. 4 Becario de Informática. Universidade de Alcalá. Departamento Didáctica. Alcalá de Henares - Madrid España.

5 Proyecto financiado por el Vicerrectorado de Planificación y Comunicación de la Universidad de Alcalá para el curso académico 2007/08.
} 


\section{Introducción: los dilemas del cambio y las continuidades y rupturas en las formas de concebir y vivir el practicum}

La transformación que se avecina exige cambios en los planes de estudio y en las metodologías de trabajo, en tanto la acción didáctica tiene que asentarse en procesos de aprendizaje más interdisciplinares, heurísticos y autónomos. Pero estos procesos no se concretarán en acciones prácticas si la mentalidad del profesorado universitario no cambia, y los estudiantes desarrollan procesos de trabajo más independientes, orientados a un aprendizaje reflexivo. En concreto, en el desarrollo del Practicum, necesitamos que los supervisores realicen un acompañamiento permanente y continuo del proceso de aprendizaje de los estudiantes, y proponer metodologías que les permitan vivenciar procesos en torno a dos dimensiones claves para el desempeño actual de la profesión docente: la necesaria interconexión teoría y práctica que los estudiantes tienen que utilizar como procedimiento vertebrador de sus procesos de aprendizaje durante las prácticas de enseñanza; y experimentar procedimientos de trabajo autónomos y reflexivos. Se trata en síntesis que los estudiantes puedan desarrollar las competencias necesarias para aprender reflexivamente de un modo continuo y permanente, y desarrollar un compromiso emocional con la función docente6.

Con estas premisas de partida realizamos un estudio orientado a conocer las percepciones y creencias que tienen los tres agentes implicados en el Practicum de la EU de Magisterio de Guadalajara, en relación con las dos cuestiones que acabamos de señalar. Con este conocimiento tuvimos un referente clave que, como punto de partida, nos sirviera para detectar y determinar las peculiaridades de la mejora desde su punto de vista, y poder diseñar y desarrollar una propuesta coherente, viable y oportuna, en la que la Web-CT nos serviría como espacio para complementar y enriquecer los procesos de indagación sobre la práctica.

Éramos conscientes de que las carencias y problemáticas en relación con el diseño y desarrollo del Practicum en los Títulos de Maestro son claramente conocidos y están bien formulados (FULLAN, 2002a; PÉREZ GÓMEZ, 1999; MONTERO, 2006; ZABALZA, 2004, 2006). Ante esta situación, acordamos que lo que nos interesaba no era, en un primer momento, conocer cómo funcionaba el Practicum para realizar una propuesta sobre cómo debería funcionar, sino cómo era vivido y experimentado por los tres agentes implicados, que

6 Para profundizar en las implicaciones que los cambios actuales tienen en la formación inicial del profesorado véase el reciente trabajo de Lourdes Montero (2006). 
desde sus subjetividades nos permitieran “escuchar, cómo lo ven y sienten ellos”. Así pues, orientamos nuestros esfuerzos a explicar las visiones, valores y motivos de los supervisores de la EU, maestros-tutores y estudiantes en relación con su experiencia subjetiva en su participación en las prácticas de enseñanza, y así poder determinar las peculiaridades del cambio. Posteriormente determinaríamos las acciones en relación con el cambio oportuno y viable, y las herramientas conceptuales y los procedimientos de trabajo en los que sustentar dicho plan7.

La primera cuestión significativa que identificamos es que el Practicum es un concepto vaciado ante el que nos resulta difícil concebir desde unos referentes integradores como espacio de deliberación y de aprendizaje para todos los implicados en su desarrollo. No hay conciencia de que el Practicum es, en primer lugar, una inmersión en una dimensión básica de la realidad educativa; la práctica, pero no la única. No es para la mayoría de los maestrostutores y supervisores de la EU, ni tampoco para los estudiantes, una forma de experimentar y vivenciar el potencial de la teoría para comprender e interpretar la calidad de lo que acontece en el aula y en el centro desde un punto de vista educativo y social.

Hay una distinción claramente dicotómica entre conocimiento formal y conocimiento práctico, en un juego en el que el realce e hincapié de la práctica es lo cotidiano que, o bien tiene unas relaciones débiles con la teoría, o bien presenta unas desconexiones evidentes (COCHRAN-SMITH; LYTLE, 2003). No predomina, en síntesis, una conciencia verdadera de que el conocimiento teórico alude también a realidades prácticas, que el mundo de la teoría también nos permite re-conocer y reflexionar sobre por qué actuamos y cómo lo hacemos; nos permite pensar, dudar e imaginar otras formas de actuar en el aula, sopesando otras alternativas de acción. Del mismo modo, hay dificultades para pensar que bajo la acción práctica hay teorías, que pueden incluso ser contrapuestas, pero que no agotan el sentido de lo que es y porqué se concreta la práctica en una acciones determinadas y no en otras. Los estudiantes se ven imposibilitados para explicitar los marcos de interpretación que dotan de sentido la práctica en la que se ven inmersos. Su capacidad para teorizar, para dotar de

7 Para Fullan (2002b, p.77) la iniciación al cambio de nuevas prácticas requiere que a nivel individual y colectivo se comprenda la conveniencia y la viabilidad de las mismas, para lo cual es necesario determinar qué cambiar y cómo hacerlo como procesos que interactúan y se dan forma el uno al otro. Para este autor. si se quiere asentar las bases para un cambio compartido y sostenido en el tiempo, tiene que desterrarse la concepción de que los cambios que se propongan son algo superior a las prácticas que se sustituyen por el mero hecho de hacer las cosas de otra manera. Y no porque desde un punto de vista objetivo y teórico no lo puedan ser, sino porque el sentido del cambio tiene un componente subjetivo irrenunciable, pero también colectivo 
significado a lo que hacen los maestros-tutores, no forma parte de sus concepciones en relación con su rol profesional.

La ambigüedad y contradicción conceptual, lo es también procedimental, y son los alumnos los que mejor expresan esta situación. Viven una situación dicotómica, un doble papel en su rol como alumnos; se mueven entre la dependencia del maestro-tutor a la dependencia del supervisor. Porque la ambigüedad y contradicción se evidencia en que no saben muy bien en algunas ocasiones qué rol desempeñar como aprendices durante su estancia en el centro porque, como reconocen abiertamente, a veces pueden sentirse perdidos, bajo un juicio y razón que no siempre son explícitos ni justos a los ojos de los alumnos. Esto les imposibilita desarrollar unos procesos de aprendizaje en el que tomen conciencia de los hallazgos que van descubriendo. La necesaria reconstrucción y enriquecimiento de su conocimiento pedagógico se ve cercenado, porque no pueden desarrollar procedimientos de trabajo que le permitan pasar de "mirar la realidad” a observarla e interpretarla. Realidad que se ve legitimada por el carácter deslocalizado del Practicum en la Escuela Universitaria.

Desde estos antecedentes, y a partir de la iniciativa de tres profesores del departamento de Didáctica de la Universidad de Alcalá desde el curso académico 2006/ 2007, se desarrolla la experiencia que se presenta a continuación cuya finalidad es aportar estrategias para la reflexión y el análisis que invitan al alumnado a vivir y sentir el hecho educativo desde múltiples miradas. Desde estas premisas de partida nace una propuesta de innovación que no es otra cosa más que una posible alternativa para la mejora de la formación inicial del profesorado que, quizá, hoy es más necesaria que nunca al encontrarnos en las "arenas movedizas” generadas por la incertidumbre ante los cambios que están por venir en el contexto universitario.

\section{El maestro reflexivo y las estrategias de aprendizaje orientadas a los procesos}

Ante la necesidad de que nuestro estudiantes vivenciaran procesos de reconstrucción del conocimiento pedagógico, y dadas las condiciones contextuales en las que se desarrollan las prácticas de enseñanza, decidimos explorar el potencial didáctico de la Web-CT.

Nos planteamos la utilización de este entorno para potenciar la tutorización por parte de los supervisores de la EU y fortalecer estrategias de aprendizaje orientadas a los procesos deliberativos y compartidos entre supervisores y estudiantes. Entendimos que la autonomía en 
el aprender y la reflexión compartida en torno a las prácticas de enseñanza se verían facilitadas a través de esta plataforma virtual.

Organizamos el trabajo en torno a lo que McKernan (2001) denomina "problemas en el currículum”. Y un problema relevante que se convierte en un desafío para nosotros es cómo el alumnado puede llevar a cabo un aprendizaje reflexivo en el practicum o lo que es lo mismo: cómo desarrollar estrategias de aprendizaje centradas en los procesos.

Para ello, partimos de una cuestión central que da sentido a la propuesta de innovación planteada:

¿Cómo promover en el alumnado un aprendizaje basado en la indagación sobre la práctica educativa, desde una conciencia crítica de lo que acontece en el centro escolar y el aula (teoría-práctica un viaje de ida y vuelta)? ¿Cómo promover una actitud reflexiva sobre el propio proceso de aprendizaje que el alumnado va realizando ante el condicionamiento de la no presencialidad en el Practicum? ¿Qué papel desempeñaría la plataforma virtual y las herramientas a ella asociadas? (MCKERNAN, 2001, p.?).

Diseñamos una propuesta de innovación basada en la enseñanza reflexiva. Distintos son los autores que en los años ochenta y noventa muestran interés por ir desplazando el énfasis en la formación del profesorado desde una perspectiva estrictamente practicista y técnica, hacia una formación más intelectual y crítica, que reconoce el conocimiento práctico como un saber que está regulado y apoyado en procesos de análisis reflexivos situados y colectivos.

El siguiente gráfico muestra la experiencia del maestro reflexivo como un hecho que tiene su fundamentación en la actividad investigadora (experiencia de indagación), puesto que propone una recogida de información de diferentes fuentes (conocimiento experiencial práctico y conocimiento teórico) basado, como mencionamos, en las vivencias personales, el conocimiento teórico y la experiencia práctica en los centros (véase figura 1) 


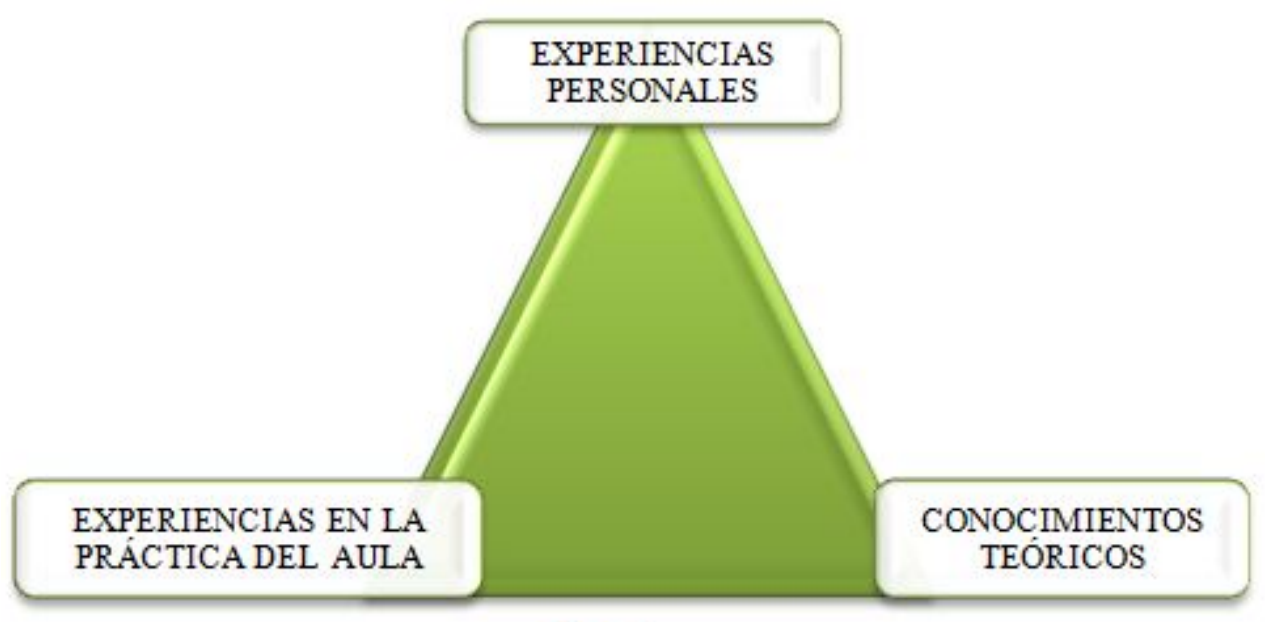

Fig. 1

La existencia de un número elevado de estudios e investigaciones realizadas sobre el tema ha ayudado a consolidar la corriente del maestro reflexivo que tomamos como referente. En este campo destacan las aportaciones de autores como Elliot, Hargreaves, Stenhouse y Schön, entre otros. Y en nuestro contexto autores como Carlos Marcelo, de la Universidad de Sevilla, Pérez Gómez en la Universidad de Málaga, y Zabalza y Montero en la Universidad de Santiago de Compostela. Los autores, más arriba citados, coinciden en un denominador común: considerar que los docentes deben adquirir su conocimiento educativo a través del nexo regulador entre sus prácticas y los análisis que hagan sobre la enseñanza, y por lo tanto sobre sus acciones y las consecuencias e implicaciones que ellas tengan.

Un elemento clave es la actitud interrogativa en la escuela, en los procedimientos utilizados para afrontar y tratar los problemas de un modo reflexivo, en definitiva, es una actitud de ser y estar como maestro. Y un gran principio que sustenta el maestro reflexivo es considerar la práctica educativa como una realidad compleja y multidimensional, determinada por diferentes factores que requiere para su intervención de una acción reflexiva y crítica, junto con una atención a las consecuencias que se pudieran derivar, como ya hemos señalado.

Para ello debemos de tener en cuenta que aprender de un modo reflexivo exige por parte del alumnado convertir su proceso de aprender en un proceso de evaluación. No es sólo el docente agente evaluador. Distintos son los autores que coinciden en señalar que la evaluación cuando se integra en el propio proceso de enseñanza-aprendizaje se convierte en factor decisivo para un aprendizaje significativo (BROCKMAN; MCGILL, 2001; MCKERNAN, 2001). Para estos autores evaluar y que el alumnado reflexione sobre su propio proceso de aprendizaje son dos caras de la misma moneda.

Por ello, se pretende que el alumno articule la reflexión sobre su aprendizaje, basado en torno a estos tres interrogantes: 
¿qué se está aprendiendo?

¿qué dificultades se van teniendo?

¿cómo se van resolviendo esas dificultades?

La vertebración de sus reflexiones en torno a estas cuestiones, son prioritarias para la inmersión del alumno en la actividad reflexiva. Esclarecedoras son las palabras de Bordas y Cabrera (2001) al respecto:

Cuando el acento se coloca en el proceso de evaluación y se imbrica con el proceso de aprendizaje, la evaluación adquiere un potencial formativo y de “empowerment” que va mucho más allá en la formación de la persona que en el mero hecho de constatar avances u objetivos conseguidos.

Desde estos planteamientos, es necesario pensar qué estrategias formativas son las más adecuadas. Para Bordas y Cabrera (2001) y McKernan (2001, p.105) el diario de aprendizaje es una estrategia formativa idónea para “fomentar la descripción, la interpretación, la reflexión y evaluación por parte del alumno”. En síntesis, desarrollar la competencia de los alumnos para reflexionar sobre su propio aprendizaje, conlleva el permitir al alumno desarrollar su autonomía, responsabilidad y participación activa en la evaluación, que en nuestro caso concreto se vertebrará a través de la elaboración de un diario personal y reflexivo en el que el alumnado vaya sistematizando pensamientos, sentimientos, dudas y valoraciones sobre qué y cómo va aprendiendo.

Estos planteamientos quedan sistematizados para nosotros en el siguiente cuadro (figura 2): 


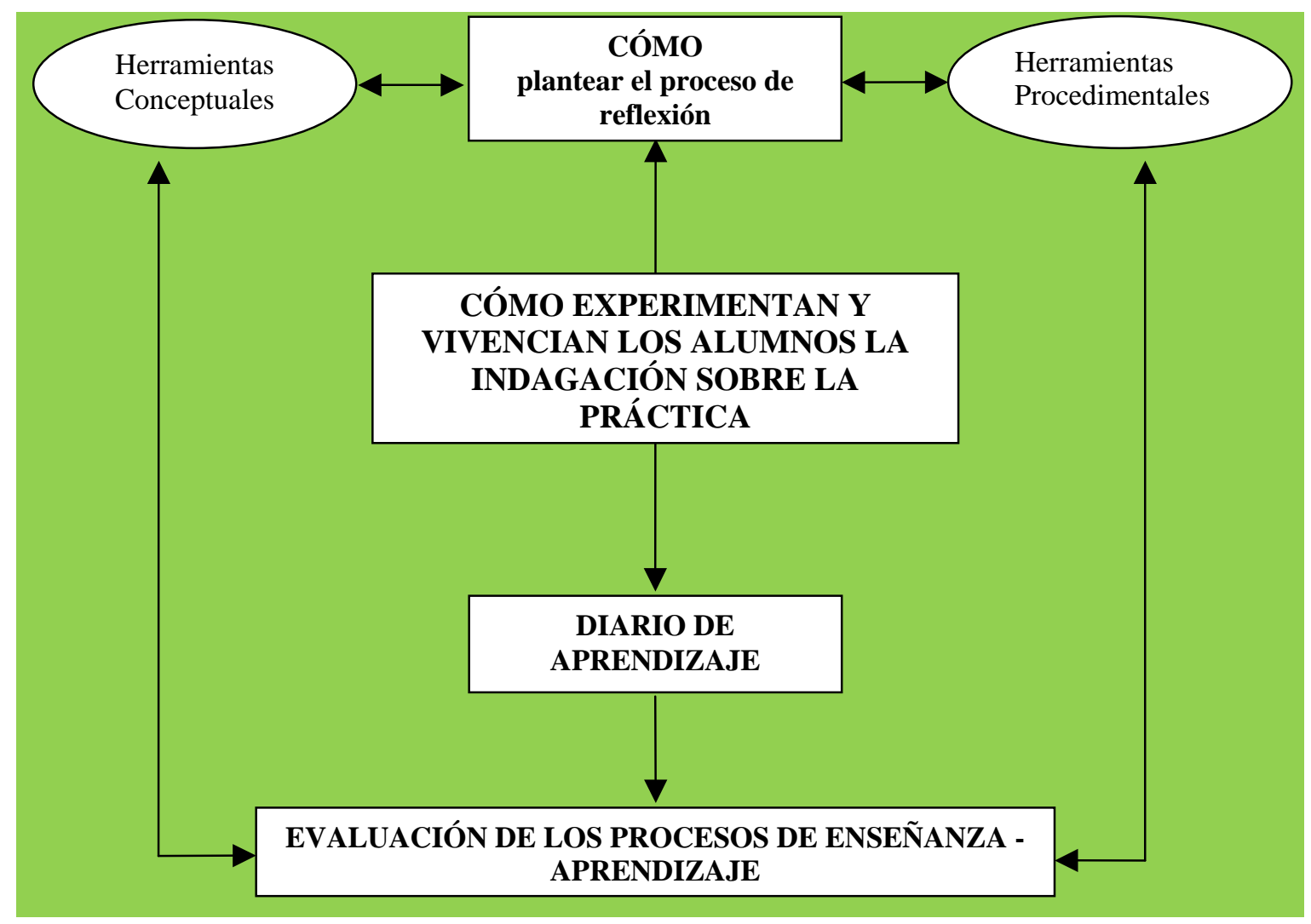

Fig. 2

Para ello, nos apoyamos en la utilización de la Web-CT de la UAH, con la clara intención de que se convierta en un espacio de intercambio de ideas, dudas, reflexiones y descubrimientos. Los foros y los blogs han sido dos grandes estrategias que nos han servido para ello. No obstante, no podemos olvidar la presencialidad como un momento y un espacio importante. Por ello, organizamos Seminarios en pequeños grupos, que complementaran los procesos de trabajo en la plataforma virtual a lo largo del desarrollo del Practicum.

Aunque para Exley y Dennick (2007) esta modalidad de enseñanza basada en pequeños grupos puede concretarse de distintos modos: seminarios, talleres, sesiones de trabajo tuteladas, o sesiones de trabajo basadas en resolución de problemas, lo importante es que un rasgo común a estas distintas formas de desarrollar la enseñanza en pequeños grupos es que el tutor trabaja con un número reducido de alumnos, y éste tiene que ofrecer oportunidades de aprendizaje en colaboración, de modo, que paulatinamente los estudiantes vayan adquiriendo autonomía, y la presencia del tutor pierda protagonismo.

\section{Presentación de la experiencia}


Desde los planteamientos de Putnam y Borko (2000), Ritcher Ershler (2001) y Korthagen et al. (2007) aprender de la práctica exige considerar que los estudiantes en formación deben realizar un aprendizaje situado, social y distribuido. Esto implica que la práctica debe ser definida y acotada por el estudiante, mediante descripciones del aula o del centro. La metodología narrativa es aquí fundamental para que el aprendiz de docente pueda situarse en un texto experiencial, que para nosotros va a tipificarse en torno a tres tipos de textos: de naturaleza autobiográfica, de carácter etnográfico de la vida en el aula, y de carácter metacognitivo sobre su proceso y resultados de aprendizaje.

Dimensiones que dan lugar a tres grandes tareas, vertebradoras de los procesos de enseñanza que girarán en torno a los niveles de experiencia que Korthagen et al. (2007) establecen como básicos para un aprendizaje reflexivo y situado en la formación inicial del profesorado a experiencia autobiográfica, la experiencia de la vida en el centro y el aula, y la experiencia personal del aprendizaje. Los objetivos y tareas propuestas quedan como aparecen en el siguiente cuadro, en el cual señalamos también los objetivos que persigue y el rol que ejerce cada uno de los implicados, así como los recursos fundamentales con los que se llevan a cabo dichas tareas. 
EXPERIENCIA PERSONAL: AUTOBIOGRAFÍA

\begin{tabular}{|c|c|c|c|}
\hline OBJETIVOS & QUÉ HACEMOS & QUIÉNES & RECURSOS \\
\hline $\begin{array}{l}\text { Autobiografía: } \\
\text { * Tomar conciencia } \\
\text { de cómo las } \\
\text { experiencias que } \\
\text { han tenido como } \\
\text { alumnos les } \\
\text { condiciona en la } \\
\text { forma de valorar } \\
\text { qué es un buen } \\
\text { profesor y qué } \\
\text { maneras de enseñar } \\
\text { son las más } \\
\text { adecuadas. }\end{array}$ & $\begin{array}{l}\text { 1.Redacción } \\
\text { individual: } \\
\text { - ¿En qué centros } \\
\text { hemos estudiado? } \\
\text { - ¿Qué recuerdo } \\
\text { tenemos de esas } \\
\text { experiencias? } \\
\text { - ¿Cómo nos } \\
\text { enseñaban? } \\
\text { - ¿Había diversidad } \\
\text { en tus clases? } \\
\text { ¿¿Qué profesores } \\
\text { recuerdas con más } \\
\text { aprecio? ¿Por qué? }\end{array}$ & $\begin{array}{l}\text { (Opcional la } \\
\text { autobiografía del } \\
\text { supervisor) }\end{array}$ & \multirow[t]{2}{*}{$\begin{array}{l}>\text { Foro de debate } \\
\\
>\text { Seminario en } \\
\text { pequeño grupo, } \\
\text { en la EU o en el } \\
\text { centro escolar }\end{array}$} \\
\hline $\begin{array}{l}\text { * Valorar si hay } \\
\text { diferencias entre el } \\
\text { contexto } \\
\text { sociocultural que } \\
\text { vivieron como } \\
\text { alumnos y el } \\
\text { momento actual en } \\
\text { relación a los } \\
\text { cambios } \\
\text { socioculturales } \\
\text { acontecidos. } \\
\text { * Qué transciendan } \\
\text { las visiones } \\
\text { convencionales de } \\
\text { la función docente. }\end{array}$ & $\begin{array}{l}\text { 2.Puesta en común: } \\
\text { - Aspectos } \\
\text { significativos y } \\
\text { relevantes de los } \\
\text { relatos } \\
\text { - Coincidencias- } \\
\text { divergencias } \\
\text { 3.Llegamos alguna } \\
\text { conclusión en } \\
\text { función de los } \\
\text { objetivos planteados } \\
\text { ¿QUIÉN SOY YO } \\
\text { COMO DOCENTE? } \\
\text { ¿QUIÉNES SON } \\
\text { MIS } \\
\text { COMPAÑEROS? }\end{array}$ & $\begin{array}{l}\text { Cada supervisor } \\
\text { con sus alumnos } \\
\text { asignados }\end{array}$ & \\
\hline $\begin{array}{l}\text { EXPERIENCIA } \\
\text { ENTORNO Y los } \\
\text { CENTRO ESCOLA }\end{array}$ & LA ESCUELA: & NALISIS SOCI & $\begin{array}{l}\text { ULTURAL DEL } \\
\text { LA VIDA DEL } \\
\text { tos, interpretamos }\end{array}$ \\
\hline
\end{tabular}




\begin{tabular}{|c|c|c|c|}
\hline \multicolumn{4}{|c|}{ (lectura y contraste teoría-práctica) y proponemos alternativas de mejora } \\
\hline OBJETIVOS & QUÉ HACEMOS & QUIÉNES & RECURSOS \\
\hline $\begin{array}{l}* \text { Recoger } \\
\text { información sobre } \\
\text { lo que ocurre en la } \\
\text { vida del centro y del } \\
\text { aula. } \\
\text { * Describir los } \\
\text { rasgos más } \\
\text { sobresalientes del } \\
\text { contexto escolar, y } \\
\text { sus implicaciones en } \\
\text { el centro escolar y el } \\
\text { aula de referencia. }\end{array}$ & $\begin{array}{l}\text { 4. Recogemos datos } \\
\text { en relación con el } \\
\text { entorno sociocultural }\end{array}$ & $\begin{array}{l}\text { Alumnado de cada } \\
\text { centro }\end{array}$ & $\begin{array}{l}>\text { Seminario en el } \\
\text { centro escolar: } \\
\text { cada } \\
\text { supervisor, con } \\
\text { sus alumnos y } \\
\text { los tutores } \\
\\
>\text { Seminario en } \\
\text { pequeño grupo, } \\
\text { en la EU o en el } \\
\text { centro escolar } \\
\\
\text { Blog personal } \\
\text { del alumno } \\
>\text { (opcional) Foro } \\
\text { de debate sobre } \\
\text { temas que } \\
\text { recogen } \\
\text { impresiones e } \\
\text { inquietudes de } \\
\text { los alumnos. }\end{array}$ \\
\hline
\end{tabular}




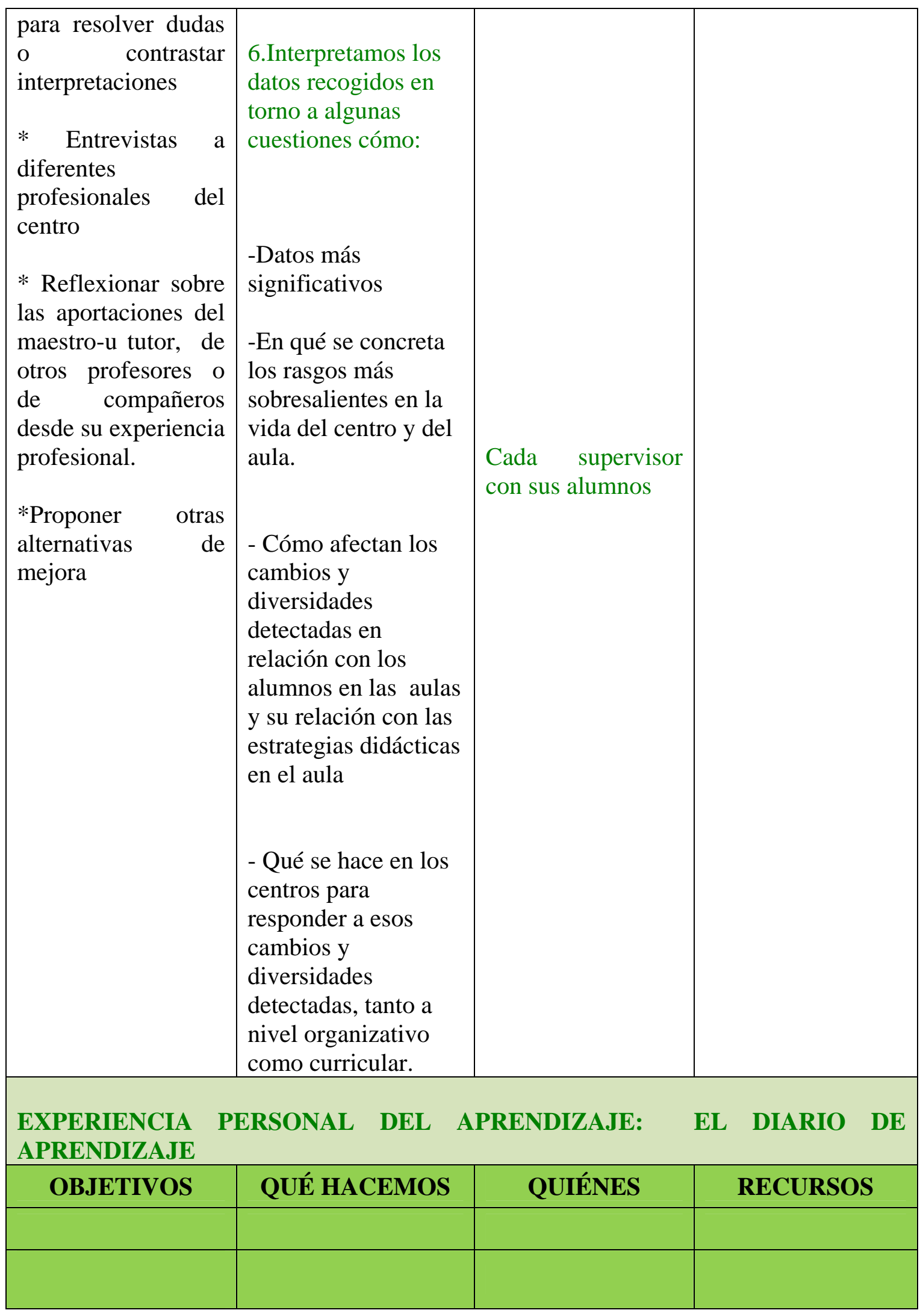


Este plan de trabajo se articula en torno a una modalidad mixta a la hora de llevar a cabo el proceso de enseñanza - aprendizaje. Por un lado, se realizan sesiones de carácter presencial cada quince días bajo la modalidad de Seminario. Con ello pretendemos poner en común dudas, dificultades, inquietudes y aspectos relevantes del aprendizaje de los alumnos. No está de más recordar, al respecto, que los procesos de aprendizaje de los alumnos tienen que ir encaminándose paulatinamente a una autoevaluación y coevaluación del aprendizaje personal y compartido.

Por otro lado, se realizan sesiones de trabajo bajo la modalidad de enseñanza virtual. Esta forma de trabajo está basada en dos tecnologías. Una la Web-CT, una plataforma de elearning que nos permite estructurar y organizar los procesos de análisis y reflexión sobre la práctica educativa a través de diversos materiales. Además, esta plataforma nos brinda la posibilidad de utilizar diversas herramientas de comunicación (correo interno y foros).

No obstante, conviene tener en cuenta que la Web-CT aunque nos permite generar un ambiente de aprendizaje bien organizado y estructurado, detectamos la necesidad de utilizar otra tecnología, lo que se conoce como blogs personales del alumno. Con ello conseguimos favorecer la presentación de las ideas y la interpretación de las conclusiones a las que van llegando los estudiantes; respetando la creatividad y la autonomía en relación con la reflexión sobre su aprendizaje, otorgando al usuario total libertad respecto al uso de cualquier material audiovisual.

\section{ESTRUCTURA Y CONEXIÓN DE LAS DOS TECNOLOGĹAS}

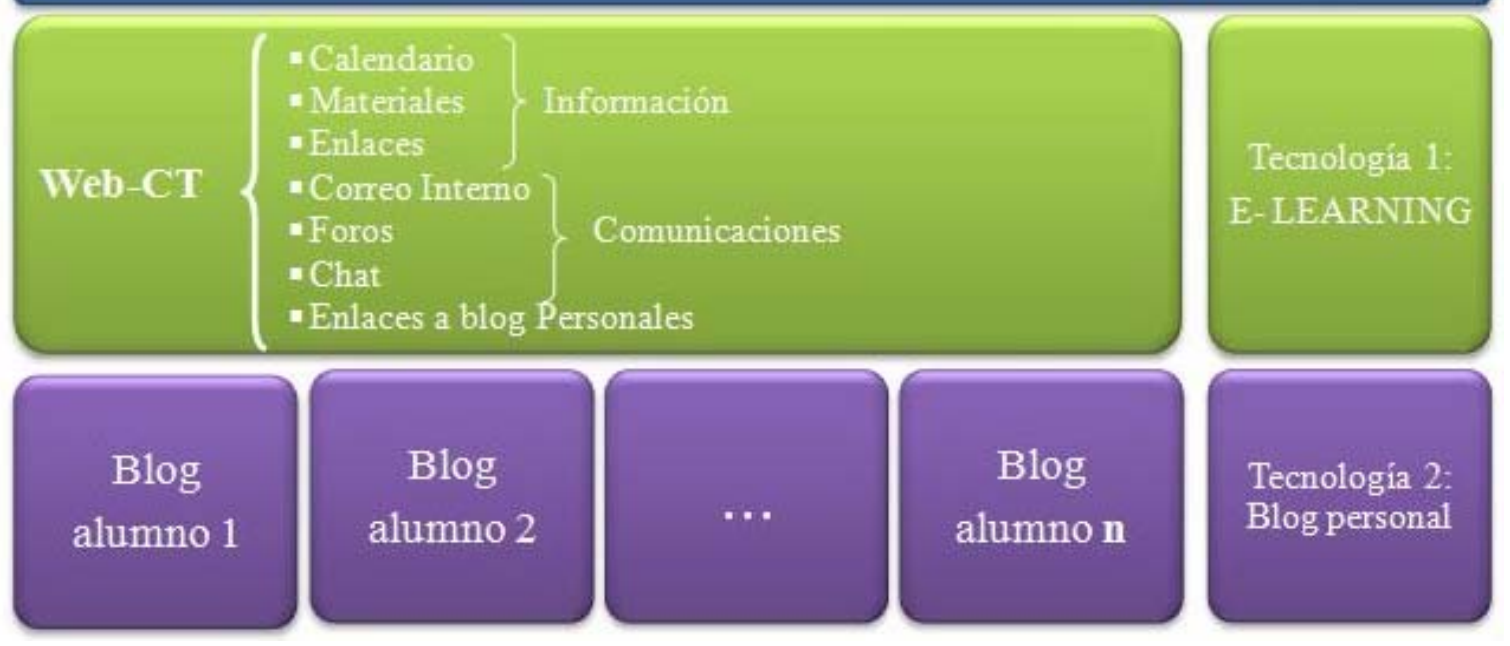


En síntesis, podemos afirmar que la utilización de la Web-CT define un espacio de comunicación e interacción entre los miembros del grupo de trabajo, orientado a desarrollar procesos de reflexión sobre la práctica de forma compartida. Concretando el papel de los foros y de los blog, consideramos que son recursos apropiados para trabajar una serie de cuestiones que se articulan en torno a tres tipos de experiencias que son objeto de análisis y reflexión por parte del alumnado. En el cuadro siguiente presentamos el papel de cada recurso electrónico y las experiencias que son objeto de análisis y reflexión por parte del alumnado.

\section{LA Web-CT DEBE CONCRETAR UN ESPACIO DE COMUNICACIÓN E INTERACCIÓN ENTRE LOS MIEMBROS DEL GRUPO DE TRABAJO, ORIENTADOS A DESARROLLAR PROCESOS DE REFLEXIÓN SOBRE LA PRÁCTICA COMPARTIDOS}

\begin{tabular}{|c|c|c|c|}
\hline & $\begin{array}{l}\text { TEMAS objeto de análisis y } \\
\text { reflexión }\end{array}$ & PARA EVIIDENCIAR & $\begin{array}{l}\text { DOCUMIENTOS } \\
\text { TRABAJO }\end{array}$ \\
\hline \multirow{3}{*}{$\begin{array}{l}\mathbf{F} \\
\mathbf{O} \\
\mathbf{R} \\
\mathrm{O}\end{array}$} & $\begin{array}{l}\text { Experiencias pasadas. } \\
\text { "Rol del alumno" }\end{array}$ & $\begin{array}{l}\text { Maestro con unas } \\
\text { preconcepciones previas que le } \\
\text { condicionan en el análisis y } \\
\text { valoración de la práctica } \\
\text { educativa }\end{array}$ & AUTOBIOGRAFÍAS \\
\hline & $\begin{array}{l}\text { Experiencias recientes en } \\
\text { los estudios Universitarios } \\
\text { de los alumnos }\end{array}$ & $\begin{array}{l}\text { La complejidad de la Teoría - } \\
\text { Práctica }\end{array}$ & \\
\hline & $\begin{array}{l}\text { Experiencias de las } \\
\text { prácticas de enseñanza }\end{array}$ & $\begin{array}{l}\text { - } \quad \text { Describir } \\
\text { - } \quad \text { Interpretar - Analizar } \\
\text { - } \quad \text { Valorar } \\
\text { lecturas científicas, el diario de } \\
\text { observación y la puesta en } \\
\text { común }\end{array}$ & $\begin{array}{llr}\text { DIARIOS } & & \text { DE } \\
\text { OBSERVACIÓN } & \text { de } & \text { la } \\
\text { práctica educativa } & & \end{array}$ \\
\hline $\begin{array}{l}\text { B } \\
\text { L } \\
\text { O } \\
\text { G }\end{array}$ & $\begin{array}{l}\text { Experiencia de aprendizaje } \\
\text { en el Practicum: el Diario } \\
\text { de mi aprendizaje }\end{array}$ & $\begin{array}{l}\text { Metacognición: } \\
\text { Autorregulación del propio } \\
\text { aprendizaje }\end{array}$ & $\begin{array}{l}\text { DIARIO DE } \\
\text { APRENDIZAJE DEL } \\
\text { ALUMNO como documento } \\
\text { que refleja todo el proceso } \\
\text { de enseñanza - aprendizaje }\end{array}$ \\
\hline
\end{tabular}




\section{Resultado obtenidos}

Los resultados obtenidos en relación a la experiencia de innovación son los siguientes:

a) La Web-CT y las aplicaciones a ellas asociadas facilitan la construcción de un contexto de comunicación para el aprendizaje reflexivo en pequeño grupo. Sin este espacio virtual, hemos constatado que pueden generarse espacios de intercambio de experiencias y puesta en común de dudas, inquietudes y dificultades, bajo una modalidad estrictamente presencial. Pero los lazos de confianza, la reflexión sobre la práctica en el aula, y la toma de conciencia de qué y cómo se está aprendiendo, se ven claramente reforzados y enriquecidos con el uso de estas herramientas. La interacción comunicativa en grupo ayuda a los estudiantes a la hora de negociar cómo elaborar una producción, al hacerla más comprensible.

Hemos constatado que los estudiantes valoran positivamente la posibilidad de acceder a las producciones del aprendizaje de sus compañeros (autobiografía, experiencias de aula y el diario de aprendizaje en los blogs). El acceso y análisis de estos productos les permite acercarse a otras estrategias de aprendizaje, así como a las dudas e inquietudes que tienen los “otros”, lo que genera un clima de autoconfianza y autoestima. Es significativo como los alumnos valoran que el “encuentro" con sus compañeros y sus “voces” en los foros, los blog y los e-mails, lo que motiva a estudiantes tímidos, inseguros o poco motivados para el aprendizaje. La retroalimentación no sólo viene del supervisor, sino de los iguales, lo que les da seguridad y una identidad de grupo.

b) La utilización de la Web-CT como espacio de comunicación e interacción entre los estudiantes, implica también un esfuerzo personal y la responsabilidad en su aprendizaje para fijarse sus tiempos y espacios de trabajo. Se mitiga de este modo una de las dificultades más importantes en el desarrollo del Practicum: la simple inmersión en los centros y el aula de referencia por parte de los alumnos, que les lleva -si no median unos procesos de aprendizaje reflexivos y más autónomos- a la adquisición de pautas de comportamiento práctico por modelado, sin una revisión y un análisis sobre la pertinencia contextual y pedagógica de las estrategias de enseñanza-aprendizaje que observan en las aulas.

Es importante apuntar, como los estudiantes, gracias al espacio virtual en el que pueden encontrarse y dialogar de modo no presencial, tienen una experiencia de aprendizaje más autónoma y consciente. Téngase en cuenta que la comunicación e interacción en diferido, elimina una de las barreras que afectan la enseñanza y el aprendizaje en esta materia, la 
deslocalización que deviene en incomunicación entre los estudiantes, y hace que el aprendizaje se haga en solitario. Sin un espacio virtual la experiencia de aprendizaje en el Practicum se focaliza en los centros escolares de referencia, con encuentros puntuales entre supervisores y estudiantes bien a través de tutoría o en sesiones plenarias de grupo. Ahora, el aprendizaje "invade" otros tiempos y otros espacios, necesarios para el enriquecimiento de los procesos de análisis, contraste e interpretación de la práctica educativa. Esta perspectiva de análisis es puesta en evidencia, en los estudios realizados por Strijbos y otros (2004 apud ONRUBIA, 2007), y Stahl (2006 apud ONRUBIA, 2007), trabajos que evidencian como un valor importante de las tecnologías el aprendizaje entre iguales, la multiplicación en el tiempo y en el espacio las posibilidades de interacción, comunicación y colaboración entre estudiantes alejados geográficamente.

c) El espacio virtual se convierte en fuente de experiencia y mediación a través de la escritura, lo que da forma, enriquece y alienta sus procesos de análisis y reflexión, y les permite valorar múltiples perspectivas de trabajar en el aula. Téngase en cuenta que las descripciones que cada alumno realiza del centro escolar y de la práctica educativa del aula de referencia, se ponen a disposición del resto a través de producciones escritas. La concreción de estas experiencias por escrito, y la ubicación y disponibilidad para el grupo de estudiantes que permite las aplicaciones asociadas a la Web-CT, no sólo les exige dar nombre a sus experiencias y reflexionar sobre ellas, también les exige nombrar con claridad y coherencia. Una de las dos tareas más complejas, y valoradas por los estudiantes como más difíciles en su experiencia de aprendizaje, pero también la más satisfactoria. Los trabajos llevados a cabo por Ritcher Ershler (2001, p.198), con profesores noveles, se orientan en esta dirección:

[...] el examen meticuloso de su práctica que precede al hecho en sí de contar historias proporciona, a los que están aprendiendo el oficio de profesores, la oportunidad de comprender sus experiencias de un modo distinto. [...] Los profesores noveles a quienes se invita a elaborar narraciones sobre el acontecer de sus aulas -y a compartirlos con otrosestán accediendo a una cultura de la enseñanza que reconoce la experiencia docente con contexto de aprendizaje.

Y ese reconocer se ve enriquecido con la diversidad de perspectivas que cada alumno aporta al resto con su Diario de Aula, de modo que pueden acercarse a la enseñanza desde una diversidad de contextos y actuaciones que enriquece su mirada.

Si bien, que los alumnos de la formación inicial se vean así mismo como aprendices “situados” en la práctica, es como hemos dicho una de las cuestiones más complejas y difíciles de conseguir. 
d) Para terminar señalar que junto a esta tarea que da lugar al "Diario de Aula", le sigue la otra de complejidad evidente, que plantea a las instituciones de formación del profesorado, uno de los restos más importantes. Y que si bien en parte, los espacios de Hemos evidenciado que la elaboración del "Diario de mi aprendizaje” exige a los estudiantes acotar y definir un texto experiencial de su propio proceso de aprender para facilitar las tareas de autoevaluación y evaluación por parte del alumno y cada supervisor. Pero estas oportunidades se ven condicionadas por el rol pasivo que como aprendices tienen los estudiantes cuando se sitúan en el aula de referencia con su maestro-tutor. Hemos constatado que a la mayoría de los alumnos les cuesta explicitar y argumentar su proceso de aprendizaje. La identificación de evidencias y conclusiones, la definición del grado de cumplimiento de los objetivos previstos teniendo en cuenta las dificultades encontradas y las soluciones adoptadas, así como la identificación de los puntos fuertes y débiles de su aprendizaje, se ven comprometidas. Y es que algunos estudiantes se sitúan en una posición de agradar al maestro-tutor, porque en nuestro caso es evaluador del trabajo realizado por escrito del alumno, cuando no mero emisor de una calificación o nota. Esto conlleva una disociación por parte del alumno, quien a veces sucumbe a un rol de sometimiento a unas reglas del juego que obstaculizan el rol de aprendiz activo y situado en la práctica.

Entendemos que esta situación ser vería mitigada si llegáramos a comprometer a los centros escolares y a los docentes, de tal modo que se crearan redes de aprendizaje profesional entre supervisores, estudiantes y maestros tutores. Miller (2001, p.145) en su trabajo en relación con el consorcio escuela y universidad aboga por un maridaje necesario como espacio de desarrollo profesional para todos los implicados. Pero como reconoce esta autora

En su raíz, un consorcio entre la escuela y la universidad es una organización precaria. Al tratar de hacer de puente entre dos culturas, no es consustancial a ninguna de las dos. Esta marginalidad, a pesar de ser difícil de manejar, es esencial para su supervivencia. No sólo lo protege de una excesiva identificación con alguna de las instituciones, sino que garantiza que las diversidades y múltiples voces serán escuchadas y valoradas.

Y en este sentido, la Web-CT y las aplicaciones a ellas asociadas podían ser un buen acicate y oportunidad para ampliar las redes de aprendizaje que hemos conformado entre supervisores de la EU de Magisterio y estudiantes en formación, a los maestros-tutores en las aulas de referencia para cada uno de nuestros alumnos. 


\title{
AN INNOVATION PROPOSAL IN THE TEACHER'S TRAINING: THE USE OF E- LEARNING AS SUPPORT TO A REFLECTIVE LEARNING
}

\begin{abstract}
In this communication we present an innovation proposal in the teacher's training based on the use of e-learning as support to a reflective learning. The context of application centres on the Practices of Education, concretly on the subject "Practicum" of the teacher's training studies, question that gives coverage to a learning based on a critical reflection on the educational practice in the school centers. The implied subjects have been two, the Practicum I and the Practicum II, and the implied pupils have been thirty four. In this document it's presented the theoretical frame and the principles that have guided the design and development of the experience. We also present the information gathered during the process and the results obtained in relation to the implications that has all this in the educational practice from the analysis of our practices of education and learning. With it we want to demonstrate the didactic possibilities of e- learning and its benefits to improve reflective and critical learning processes in the teacher's training.
\end{abstract}

KEYWORDS: e- learning, teacher's training, educational practices, comprehension and reflective teaching learning.

\section{REFERENCIAS}

BORDAS, M. I.; CABRERA, F. A. Estrategias de evaluación de los aprendizajes centrados en el proceso. Revista Española de Pedagogía, Barcelona, año LIX, n.218, p.25-48, eneroabr. 2001.

BROCKMAN G.; MCGILL, H. El aprendizaje crítico en la enseñanza superior. Madrid: Morata, 2001.

COCHRAN-SMITH, M.; LYTLE, S. Más allá de la certidumbre: adoptar una actitud indagadora sobre la práctica. In: LIEBERMAN, A.; MILLER, L. (Ed.). La indagación como base de la formación del profesorado y la mejora de la educación. Barcelona: Octaedro, 2003. p.65-79.

EXLEY, K.; DENNICK, R. Enseñanza en pequeños grupos en educación superior. Madrid: Nancea, 2007.

FULLAN, M. Las fuerzas del cambio: explorando las profundidades de la reforma educativa. Madrid: Akal, 2002a.

Los nuevos significados del cambio en la educación. Barcelona: Octaedro, 2002b. 
GIMENO SACRISTÁN, J. El alumno como invención. Madrid: Morata, 2003.

KORTHAGEN, A. et al. Learning from practice: a comenius 2.1. project. Disponible en: <http://www2.ivlos.uu.nl/comenius/downloads/Grundlegende\%20Konzepte.pdf>. Acceso en: 14 feb. 2007.

MARCELO, C. La función docente. Madrid: Ed. Síntesis, 2001.

MCKERNAN, J. Investigación-acción y curriculum. Madrid: Morata, 2001.

MILLER, L. El consorcio entre la escuela y la universidad como lugar de encuentro para el desarrollo profesional. In: LIEBERMAN, A.; MILLER, L (Ed.) La indagación como base de la formación del profesorado y la mejora de la educación. Barcelona: Octaedro, 2001. p.129-146.

MONTERO, L. Profesores y profesoras en un mundo cambiante: el papel clave de la formación inicial. Revista de Educación, Madrid, n.340, p.66-86, mayo/agosto 2006.

ONRUBIA, J. Las tecnologías de la información y la comunicación como instrumento de apoyo a la innovación de la docencia universitaria. Revista Interuniversitaria de Formación del Profesorado, Zaragoza, v.21, n.1, p.21-36, 2007.

PÉREZ GÓMEZ, A. I. El practicum de enseñanza y la socialización profesional de los futuros docentes. In: PÉREZ GÓMEZ, A. I. et al. (Coord.). Desarrollo profesional del docente: política, investigación y práctica. Madrid: Akal, 1999. p.636-660.

PUTNAM, R. T.; BORKO, H. ¿What do new views of knowledge and thinking have to say about research on teacher learning? Educational Researcher, London, v.29, n.1, p.4-16, 2000.

RITCHER ERSHLER, A. La narrativa como texto experiencial: incluirse en el texto. In: LIEBERMAN, A.; MILLER, L. (Ed.) La indagación como base de la formación del profesorado y la mejora de la educación. Barcelona: Octaedro, 2001. p. 193-208.

ZABALZA, M. Buscando una nueva hoja de ruta en la formación del profesorado. Revista de Educación, Madrid. n.340, p.51-55, mayo/agosto, 2006.

Condiciones para el desarrollo del practicum. Revista de Curriculum y Formación del Profesorado, Granada, v.8, n.2, p.1-22, 2004. 